\title{
Characteristics of Extremely Early-Onset Pouchitis after Proctocolectomy with Ileal Pouch-Anal Anastomosis
}

\author{
Yoshiki Okita • Toshimitsu Araki • Koji Tanaka • \\ Tadanobu Shimura $\cdot$ Mikio Kawamura • \\ Hiroyuki Fujikawa • Mikihiro Inoue • Yasuhiro Inoue • \\ Keiichi Uchida • Yasuhiko Mohri • Masato Kusunoki
}

Received: 4 July 2012 / Accepted: 3 December 2012 / Published online: 20 December 2012

(C) 2012 The Author(s). This article is published with open access at Springerlink.com

\begin{abstract}
Objective This study aims to evaluate the clinical characteristics of patients with postoperative ulcerative colitis developing extremely early-onset pouchitis within 4 weeks of restoration of gastrointestinal continuity.

Material and Methods This retrospective study involved 225 consecutive patients from January 2000 to May 2011 who underwent ileal pouch-anal anastomosis for ulcerative colitis with a minimum follow-up of 12 months. Extremely earlyonset pouchitis was defined as pouchitis developing within 4 weeks of restoration of gastrointestinal continuity. Patients with pouchitis were divided into extremely early-onset pouchitis and non-extremely early-onset pouchitis groups for comparison. Results Of 212 patients who met the inclusion criteria, 62 had pouchitis (29.2\%). Eight (3.8\%) patients developed extremely early-onset pouchitis. There was a significant difference in the dosage of steroid per month just before colectomy between the two groups $(P=0.039)$. The modified Pouchitis Disease Activity Index score for patients with extremely early-onset pouchitis was higher than in patients with non-extremely early-onset pouchitis $(P=0.0009)$. The occurrence of extremely early-onset pouchitis was associated with the development of chronic pouchitis $(P=0.0056)$.

Conclusion Extremely early-onset pouchitis may be related to high steroid dosages before colectomy and may be more severe than pouchitis that occurs $>4$ weeks after restoration of gastrointestinal continuity.
\end{abstract}

Keywords Ulcerative colitis $\cdot$ Corticosteroids $\cdot$ Pouchitis

\section{Introduction}

Pouchitis, a nonspecific inflammatory condition of the ileal pouch reservoir, is the most common long-term complication in patients who have undergone ileal pouch-anal anastomosis (IPAA), and it significantly affects patients' quality of life. ${ }^{1}$

The reported cumulative risk of developing pouchitis varies widely, ranging from 15 to $50 \%$ in patients with ulcerative colitis (UC), and may be even higher $^{2-5}$. It occurs

Y. Okita $(\bowtie) \cdot$ T. Araki $\cdot$ K. Tanaka $\cdot$ T. Shimura $\cdot$ M. Kawamura $\cdot$

H. Fujikawa $\cdot$ M. Inoue $\cdot$ Y. Inoue $\cdot$ K. Uchida $\cdot$ Y. Mohri •

M. Kusunoki

Departments of Gastrointestinal and Pediatric Surgery, Division of

Reparative Medicine, Institute of Life Sciences, Mie University

Graduate School of Medicine, 2-174 Edobashi,

Tsu, Mie 514-8507, Japan

e-mail: nyokkin@clin.medic.mie-u.ac.jp most commonly within the first 6 months after IPAA and develops in $7-15 \%$ of patients. ${ }^{2,4,6-8}$

Patients with pouchitis have a wide range of clinical presentations. ${ }^{9}$ To the best of our knowledge, the relationship between onset days and clinical characteristics of pouchitis has not been fully examined in previous studies. We analyzed the histories of patients with UC who developed pouchitis within 4 weeks of ileostomy closure. Identification of the clinical characteristics of patients with extremely early-onset pouchitis (EEOP) is important for recognition of early postoperative complications and assurance of effective management. We present the characteristics of eight patients with EEOP.

\section{Material and Methods}

Study Population

This retrospective study evaluated the clinical characteristics of EEOP by reviewing Mie University's pouchitis database. 
All 225 consecutive patients from January 2000 to May 2011, who underwent IPAA for UC with a minimum follow-up of 12 months in our unit, were enrolled. Patients were reviewed at 3-month intervals for the first postoperative year and every 6 months or as needed, thereafter.

\section{Standard Operation}

The standard operation was a mucosal restorative proctocolectomy with hand-sewn IPAA using an $18 \mathrm{~cm}$ twolimbed J-shaped ileal pouch. Of the 225 patients, 223 patients underwent hand-sewn IPAA, with two patients with anal sphincter dysfunction undergoing stapled IPAA. Closure of the ileostomy was performed approximately 3 months after IPAA if an intact pouch without anastomotic leakage was confirmed just before ileostomy closure. We routinely examined ileal pouch-anal anastomosis using digital examination, manometry, and defecography before ileostomy closure. Our surgeons performed bougienage just before ileostomy closure for the patients with anastomotic stricture, and/or patients repeated self-bougienage after ileostomy closure.

\section{Diagnosis of Pouchitis}

All patients with pouchitis were diagnosed using a combination of clinical and endoscopic techniques. Biopsies were not routinely taken. Pouchitis was defined as a modified Pouchitis Disease Activity Index (PDAI) score of $\geq 5$ points. ${ }^{10}$ We performed endoscopy for all of the patients with suspected pouchitis. Endoscopic findings included edema, granularity, friability, loss of vascular pattern, mucoid exudate, and ulceration, but excluded staple-line ulcer. We performed digital examination of the ileal pouch-anal anastomosis before pouchoscopy. Secondary pouchitis (e.g., anastomotic stricture, pelvic sepsis, pouch ischemia, Clostridium difficile infection, cytomegalovirus infection and regular use of nonsteroidal anti-inflammatory drugs), and Crohn's disease were actively excluded before diagnosing pouchitis. The onset days of pouchitis were defined as the time period from restoration of gastrointestinal continuity to endoscopic diagnosis at the first episode. EEOP was defined as pouchitis that developed within 4 weeks of restoration of gastrointestinal continuity. Non-EEOP was defined as pouchitis that developed $>4$ weeks after restoration of gastrointestinal continuity. Absence of pouchitis was defined as a modified PDAI score of $\leq 4$ points. Pouchitis was classified as acute and chronic pouchitis. ${ }^{11}$ Acute pouchitis was defined as $<4$-week duration of symptoms and chronic pouchitis as $>4$-week duration. Antibiotic-responsive pouchitis was defined as an episode of pouchitis that responded to a 2week course of antibiotics. Antibiotic-dependent pouchitis was defined as disease requiring long-term, continuous antibiotic therapy to maintain remission. Antibiotic-refractory pouchitis fails to respond to antibiotics and typically requires oral or topical 5-aminosalicylates, corticosteroid therapy, or oral immunomodulator therapy. ${ }^{11}$ Antibioticdependent and antibiotic-refractory phenotypes were categorized as chronic pouchitis. We divided patients with pouchitis into EEOP and non-EEOP groups according to onset days, for comparison.

Possible factors related to the development of EEOP included: gender (male), age at initial surgery, $>32$ years; disease duration of $<6$ years; type of disease (acute fulminating vs. relapsing-remitting vs. chronic continuous); extent of colitis (left-sided colitis); severity of colitis (severe); extraintestinal manifestation; total dosage of steroid before colectomy of $>8 \mathrm{~g}$; dosage of steroid per month just before surgery $>600 \mathrm{mg}$; use of immunomodulators; emergency or urgent operation at first operation; operation stages (two vs. three stages); and pelvic sepsis. These factors were assessed using univariate and multivariate logistic regression analyses to determine the significant factors related to EEOP among patients with pouchitis. We justified age at initial surgery of 32 years; disease duration of 6 years; total dosage of steroid before surgery of $8 \mathrm{~g}$; and dosage of steroid per month just before surgery of $600 \mathrm{mg}$ as cutoff values using receiver-operator characteristic curves.

\section{Statistical Analysis}

Quantitative data were expressed as mean \pm SD (range). Statistical analysis was performed using the chi-square test or Fisher's exact test and the Mann-Whitney $U$ test for quantitative and qualitative variables using JMP version 5 (SAS Institute, Inc., Cary, NC, USA). All variables associated with EEOP with $P<0.4$ in univariate analysis of categorical data were examined consecutively using multivariate analysis logistic regression. A $P$ value of $<0.05$ was considered significant.

\section{Results}

Of the 225 patients, 13 with an ileostomy or who had undergone ileostomy reconstruction for pelvic sepsis or perianal fistula were excluded. Of the 212 patients who met the inclusion criteria, 62 had pouchitis $(29.2 \%)$. Neither of the two patients who underwent stapled IPAA developed cuffitis or pouchitis. All of the patients with pelvic sepsis and perianal fistula were radiographically confirmed to be improved before ileostomy closure. No patients with pouchitis had concurrent anastomotic stricture when they developed pouchitis. Eight (3.8 \%) patients developed EEOP. The median number of days between restoration of gastrointestinal continuity and first pouchitis onset was 587.5 days 
J Gastrointest Surg (2013) 17:533-539

535

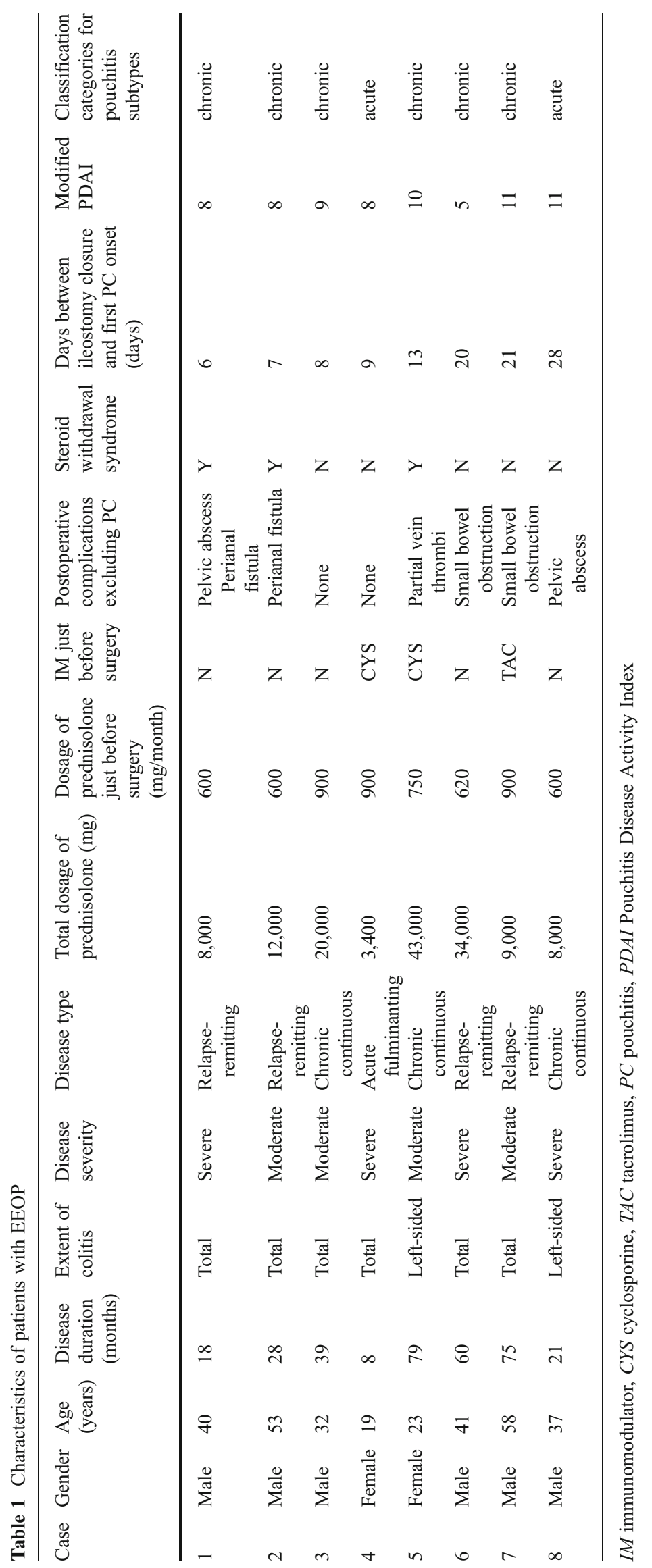

Springer 
Table 2 The clinical characteristics of the non-EEOP and EEOP patients

\begin{tabular}{|c|c|c|c|}
\hline Factors & $\begin{array}{l}\text { Non-EEOP } \\
n=54\end{array}$ & $\begin{array}{l}\text { EEOP } \\
n=8\end{array}$ & $P$ \\
\hline Gender (male/female) & $30 / 24$ & $6 / 2$ & 0.45 \\
\hline Age at initial surgery (years) & $32 \pm 15$ & $38 \pm 13$ & 0.24 \\
\hline Disease duration (years) & $7.5 \pm 6.9$ & $5.5 \pm 6.2$ & 0.28 \\
\hline Type of disease (acute fulminating/relapse-remitting/chronic continuous) & $5 / 38 / 11$ & $1 / 4 / 3$ & 0.49 \\
\hline Extent of colitis (left-sided colitis/total colitis) & $7 / 47$ & $2 / 6$ & 0.33 \\
\hline Severity of colitis (severe/moderate/mild) & $11 / 32 / 11$ & $2 / 5 / 1$ & 0.86 \\
\hline Extraintestinal manifestation $(\mathrm{Y} / \mathrm{N})$ & $2 / 52$ & $0 / 8$ & 1.00 \\
\hline Total dosage of steroid before surgery $(\mathrm{g})$ & $19.1 \pm 19.6$ & $17.6 \pm 13.8$ & 0.90 \\
\hline Dosage of steroid per month just before surgery (mg) & $518 \pm 450$ & $734 \pm 146$ & 0.03 \\
\hline Immunomodulator $(\mathrm{Y} / \mathrm{N})$ & $22 / 32$ & $4 / 4$ & 0.71 \\
\hline Emergency or urgent operation at first operation $(\mathrm{Y} / \mathrm{N})$ & $11 / 43$ & $1 / 7$ & 0.59 \\
\hline Operation stages (one stage/two stage/three stage) & $3 / 37 / 14$ & $0 / 7 / 1$ & 0.52 \\
\hline Postoperative pelvic abscess $(\mathrm{Y} / \mathrm{N})$ & $8 / 46$ & $2 / 6$ & 0.60 \\
\hline
\end{tabular}

EEOP extremely early-onset pounchitis, non-EEOP pouchitis excluding extremely early-onset pouchitis

(range, 6-3,352 days). Table 1 shows the characteristics of patients with EEOP. Six male and two female patients with a mean age of $38 \pm 13$ years (19-58 years) developed EEOP. Seven of eight patients had total colitis, and four of eight had severe colitis. The average cumulative dosage of steroid administered before colectomy in the patients with EEOP was $17.6 \mathrm{~g}$. The average dosage of steroid per month just before colectomy in patients with EEOP was $734 \mathrm{mg}$.

Of the eight patients who developed EEOP, two had pelvic sepsis, two had perianal fistula, two had small bowel obstruction, and one had portal vein thrombi after IPAA. All of the pelvic sepsis and small bowel obstruction patients improved before ileostomy closure. Two patients developed perianal fistula 3 and 5 months after ileostomy closure, respectively, and seton drainage improved the perianal fistulas. One patient with portal vein thrombi required warfarin therapy. None of the patients with EEOP had concurrent postoperative complications when they developed pouchitis except the patient with portal vein thrombi.

The clinical characteristics of the non-EEOP and EEOP patients are shown in Table 2. Univariate analysis revealed a significant difference in the dosage of steroid per month just before colectomy between the two groups $(P=0.025)$. There was no difference in gender, age at initial surgery, disease duration, type of disease, extent of colitis, severity of colitis, extraintestinal manifestation, cumulative dosage of steroid before colectomy, use of immunomodulators, emergency or urgent operation at first operation, operation stages, or pelvic sepsis between patients
Table 3 The results of univariate analysis of categorical data

\begin{tabular}{llll}
\hline Factors & Odds ratio & 95\%CI & $P$ \\
\hline Gender (male) & 1.35 & $0.85-2.15$ & 0.45 \\
Age at initial surgery ( $>32$ years) & 4.04 & $0.75-21.89$ & 0.13 \\
Disease duration ( $<6$ years) & 1.79 & $0.39-8.27$ & 0.45 \\
Type of disease (chronic continuous) & 2.35 & $0.48-11.35$ & 0.36 \\
Type of disease (relapse-remitting) & 0.42 & $0.09-1.89$ & 0.42 \\
Type of disease (acute fulminating) & 1.40 & $0.14-13.8$ & 0.58 \\
Extent of colitis (left-sided colitis) & 2.24 & $0.37-13.36$ & 0.33 \\
Severity of colitis (severe) & 1.30 & $0.23-7.36$ & 0.67 \\
Total dosage of steroid before surgery $(>8$ g) & 1.08 & $0.24-4.76$ & 1.00 \\
Dosage of steroid per month just before surgery (>600 mg) & 4.12 & $0.88-19.82$ & 0.10 \\
Use of immunomodulator (Y) & 1.45 & $0.33-6.44$ & 0.71 \\
Emergency or urgent operation at first operation (Y) & 0.56 & $0.06-5.00$ & 1.00 \\
Operation stages (two stage) & 3.22 & $0.37-28.24$ & 0.42 \\
Operation stages (three stage) & 0.41 & $0.05-3.57$ & 0.67 \\
Postoperative pelvic abscess (Y) & 1.22 & $0.13-11.27$ & 1.00 \\
\hline
\end{tabular}


Table 4 Multivariate analysis showing the dosage of steroid per month just before surgery

\begin{tabular}{lccc}
\hline & Odds ratio & $95 \% \mathrm{CI}$ & $P$ \\
\hline Age at initial surgery (>32 years) & 3.60 & $0.66-25.55$ & 0.14 \\
Type of disease (chronic continuous) & 4.69 & $0.63-44.83$ & 0.13 \\
Extent of colitis (left-sided colitis) & 1.58 & $0.08-6.05$ & 0.67 \\
Dosage of steroid per month just before surgery $(>600 \mathrm{mg})$ & 6.26 & $1.09-52.78$ & 0.039 \\
\hline
\end{tabular}

with non-EEOP and EEOP. The results of univariate analysis of categorical data did not reveal a significant difference between the two groups (Table 3). Multivariate analysis was performed using four factors: age at initial surgery $>32$ years, type of disease (chronic continuous), extent of colitis (left-sided colitis), or dosage of steroid per month just before surgery $>600 \mathrm{mg}$. Multivariate analysis showed that the dosage of steroid per month just before surgery was significantly associated with EEOP $(P=$ 0.039; Table 4).

Comparisons of severity between patients with nonEEOP and EEOP are shown in Table 5. The median number of days between restoration of gastrointestinal continuity and first pouchitis onset was 737 days in patients with non-EEOP and 11 days in patients with EEOP. The modified PDAI score (including clinical criteria and endoscopic criteria) at first onset in patients with EEOP was higher than in patients with non-EEOP $(P=0.0009)$. The occurrence of EEOP was significantly associated with the development of chronic pouchitis at first onset compared with nonEEOP patients $(P=0.0056)$. None of the patients with pouchitis required permanent ileostomy for refractory pouchitis.

\section{Discussion}

Abdelrazeq et al. ${ }^{12}$ reported that the first episode of pouchitis occurred within 6 months of restoration of gastrointestinal continuity in $45 \%$ of patients who developed pouchitis. The mean interval from the beginning of pouch function to the occurrence of the first episode of pouchitis was 5 months (range, 0.5-48 months). Other previous reports indicated that the incidence of the first attack of pouchitis was highest during the first 6 months after restoration of gastrointestinal continuity, that the mean interval from the beginning of pouch function to the first episode of pouchitis was
5-17 months, and that the range of onset was 2 days to 156 months. ${ }^{5,6,13-15}$ However, to the best of our knowledge, no previous studies of EEOP have fully examined the relationship between onset days and clinical characteristics of pouchitis.

Several studies have reported an increased incidence of pouchitis in patients with extraintestinal manifestations, primary sclerosing cholangitis, and genetic factors (IL-1ra, NOD2/CARD 15, and TNF genes). ${ }^{2,4-6,11,13,16-18}$ Recent studies have reported a relationship between the occurrence of pouchitis and preoperative steroid use. ${ }^{13,19,20}$ Fleshner et al. ${ }^{13}$ reported that clinical factors associated with the development of acute pouchitis include the use of a steroid before colectomy, although they did not describe the use or dose of the steroid in detail. Kalkan et al. ${ }^{19}$ reported that a high preoperative Disease Activity Index score and steroid dependency are independent risk factors for the development of pouchitis. They suggested that more severe disease activity in the preoperative period may pose an increased risk for the development of pouchitis. Nakamura et al. ${ }^{20}$ reported that many joint symptoms could be the result of alterations in the steroid dose taken by patients prior to surgery and that the presence of joint symptoms during steroid tapering is a significant risk factor for the development of pouchitis. In our study, there was a significant difference in the dosage of steroid per month just before colectomy between patients with EEOP and with non-EEOP. The mechanism of how steroid use relates to EEOP remains unclear. Although patients with more aggressive disease may have a higher incidence of pouchitis after surgery, this trend was not seen in patients taking other, more potent immunosuppressive agents, before IPAA. ${ }^{13}$ Suzuki et al. ${ }^{21}$ reported a relationship between the modified PDAI score at the first diagnosis of pouchitis and the subsequent clinical course. Patients with chronic, frequent, and antibiotic-dependent or refractory pouchitis had higher
Table 5 Comparisons of severity between patients with nonEEOP and EEOP

EEOP extremely early-onset pouchitis, non-EEOP pouchitis excluding extremely early-onset pouchitis, PDAI Pouchitis Disease Activity Index

\begin{tabular}{llll}
\hline Factors & Non-EEOP $(\mathrm{n}=54)$ & EEOP & $P$ \\
\hline Modified PDAI & $6.1 \pm 1.5$ & $8.8 \pm 2.0$ & 0.0009 \\
Clinical criteria & $2.9 \pm 0.8$ & $4.0 \pm 0.9$ & 0.0031 \\
Endoscopic criteria & $3.2 \pm 1.0$ & $4.8 \pm 1.4$ & 0.0035 \\
Classification categories for pouchitis & $42 / 12$ & $2 / 6$ & 0.0056 \\
$\quad$ subtypes (acute/chronic) & & & \\
\hline
\end{tabular}


modified PDAI scores than did patients with acute, infrequent, and antibiotic-responsive pouchitis. However, they did not describe the relationship between onset days and modified PDAI score. In this study, patients with EEOP were associated with higher modified PDAI score and the development of chronic pouchitis compared with nonEEOP patients. There is a possibility that patients with EEOP could be diagnosed earlier because more severe symptomatic pouchitis is easier to detect.

The limits of the present study relate to the small numbers in the two groups. Hoda et al. ${ }^{22}$ showed that patients with postoperative complications, specifically anastomotic complications, were more likely to develop chronic pouchitis. Because of the small sample size, this study may not have shown differences in pelvic sepsis between non-EEOP and EEOP, which was significantly related to chronic pouchitis. In addition, diagnosing pouchitis without biopsy may lead to diagnostic bias. New criteria for the diagnosis of pouchitis were proposed in Japan. These criteria are based on clinical symptoms and endoscopic findings that are clearly categorized in the atlas and exclude infectious enteritis, anastomotic insufficiency, pelvic infection, anal dysfunction, and Crohn's disease. Advantages of the new criteria include ease of bedside diagnosis without the calculation of points required by the other criteria for pouchitis. ${ }^{23}$ We diagnosed pouchitis based on clinical symptoms and endoscopic findings in our patients.

\section{Conclusion}

The present study is the first to report the characteristics of EEOP occurring within 4 weeks of restoration of gastrointestinal continuity. The results show that EEOP has characteristics that differ from those of non-EEOP. Some studies have reported an increased incidence of pouchitis in patients who were administered steroids. In particular, the dosage of steroid per month just before colectomy may affect the early occurrence of pouchitis. This study suggests that EEOP may be related to a high preoperative dosage of steroid and may be more severe than pouchitis that occurs $>4$ weeks after restoration of gastrointestinal continuity. Recognition of the clinical characteristics of EEOP may ensure effective management for patients with $\mathrm{UC}$ in the early postoperative period. Regardless of the reasons why an increased incidence of pouchitis was observed in patients who were administered steroids, further studies on this observation may provide valuable insights into the pathogenesis of pouchitis.

Open Access This article is distributed under the terms of the Creative Commons Attribution License which permits any use, distribution, and reproduction in any medium, provided the original author(s) and the source are credited.

\section{References}

1. Shen B, Fazio VW, Remzi FH, Delaney CP, Bennett AE, Achkar JP, Brzezinski A, Khandwala F, Liu W, Bambrick ML, Bast J, Lashner B. Comprehensive evaluation of inflammatory and noninflammatory sequelae of ileal pouch-anal anastomoses. Am J Gastroenterol 2005; 100: 93-101.

2. Hurst RD, Molinari M, Chung TP, Rubin M, Michelassi F. Prospective study of the incidence, timing and treatment of pouchitis in 104 consecutive patients after restorative proctocolectomy. Arch Surg 1996; 131: 497-500; discussion 501-492.

3. Simchuk EJ, Thirlby RC. Risk factors and true incidence of pouchitis in patients after ileal pouch-anal anastomoses. World J Surg 2000; 24: 851-856.

4. Stahlberg D, Gullberg K, Liljeqvist L, Hellers G, Lofberg R. Pouchitis following pelvic pouch operation for ulcerative colitis. Incidence, cumulative risk, and risk factors. Dis Colon Rectum 1996; 39: 1012-1018.

5. Penna C, Dozois R, Tremaine W, Sandborn W, LaRusso N, Schleck C, Ilstrup D. Pouchitis after ileal pouch-anal anastomosis for ulcerative colitis occurs with increased frequency in patients with associated primary sclerosing cholangitis. Gut 1996; 38: 234-239.

6. Lohmuller JL, Pemberton JH, Dozois RR, Ilstrup D, van Heerden J. Pouchitis and extraintestinal manifestations of inflammatory bowel disease after ileal pouch-anal anastomosis. Ann Surg 1990; 211: 622-627; discussion 627-629.

7. Mignon M, Stettler C, Phillips SF. Pouchitis—a poorly understood entity. Dis Colon Rectum 1995; 38: 100-103.

8. Moskowitz RL, Shepherd NA, Nicholls RJ. An assessment of inflammation in the reservoir after restorative proctocolectomy with ileoanal ileal reservoir. Int J Colorectal Dis 1986; 1: 167-174.

9. Yu ED, Shao Z, Shen B. Pouchitis. World J Gastroenterol 2007; 13: 5598-5604.

10. Shen B, Achkar JP, Connor JT, Ormsby AH, Remzi FH, Bevins CL, Brzezinski A, Bambrick ML, Fazio VW, Lashner BA. Modified pouchitis disease activity index: a simplified approach to the diagnosis of pouchitis. Dis Colon Rectum 2003; 46: 748-753.

11. Pardi DS, D'Haens G, Shen B, Campbell S, Gionchetti P. Clinical guidelines for the management of pouchitis. Inflamm Bowel Dis 2009; 15: 1424-1431.

12. Abdelrazeq AS, Kandiyil N, Botterill ID, Lund JN, Reynolds JR, Holdsworth PJ, Leveson SH. Predictors for acute and chronic pouchitis following restorative proctocolectomy for ulcerative colitis. Colorectal Dis 2008; 10: 805-813.

13. Fleshner P, Ippoliti A, Dubinsky M, Ognibene S, Vasiliauskas E, Chelly M, Mei L, Papadakis KA, Landers C, Targan S. A prospective multivariate analysis of clinical factors associated with pouchitis after ileal pouch-anal anastomosis. Clin Gastroenterol Hepatol 2007; 5: 952-958; quiz 887.

14. Okon A, Dubinsky M, Vasiliauskas EA, Papadakis KA, Ippoliti A, Targan SR, Fleshner PR. Elevated platelet count before ileal pouchanal anastomosis for ulcerative colitis is associated with the development of chronic pouchitis. Am Surg 2005; 71: 821-826.

15. Ikeuchi H, Nakano H, Uchino M, Nakamura M, Yanagi $H$, Noda M, Yamamura T. Incidence and therapeutic outcome of pouchitis for ulcerative colitis in Japanese patients. Dig Surg 2004; 21: 197-201.

16. Achkar JP, Al-Haddad M, Lashner B, Remzi FH, Brzezinski A, Shen B, Khandwala F, Fazio V. Differentiating risk factors for acute and chronic pouchitis. Clin Gastroenterol Hepatol 2005; 3: 60-66.

17. Hata K, Watanabe T, Shinozaki M, Nagawa H. Patients with extraintestinal manifestations have a higher risk of developing 
pouchitis in ulcerative colitis: multivariate analysis. Scand J Gastroenterol 2003; 38: 1055-1058.

18. Wasmuth HH, Trano G, Endreseth BH, Wibe A, Rydning A, Myrvold HE. Primary sclerosing cholangitis and extraintestinal manifestations in patients with ulcerative colitis and ileal pouchanal anastomosis. J Gastrointest Surg 2010; 14: 1099-1104.

19. Kalkan IH, Dagli U, Onder FO, Tunc B, Oztas E, Ulker A, Sasmaz N (2012) Evaluation of preoperative predictors of development of pouchitis after ileal-pouch anastomosis in ulcerative colitis. Clin Res Hepatol Gastroenterol 36(6):622-627.

20. Nakamura M, Ikeuchi $H$, Nakano H, Uchino M, Noda M, Yanagi H, Yamamura T. Postoperative joint symptoms: a risk factor for pouchitis in ulcerative colitis patients. Dig Surg 2006; 23: 60-64.
21. Suzuki H, Ogawa H, Shibata C, Haneda S, Watanabe K, Takahashi K, Funayama Y, Sasaki I. The long-term clinical course of pouchitis after total proctocolectomy and IPAA for ulcerative colitis. Dis Colon Rectum 2012; 55: 330-336.

22. Hoda KM, Collins JF, Knigge KL, Deveney KE. Predictors of pouchitis after ileal pouch-anal anastomosis: a retrospective review. Dis Colon Rectum 2008; 51:554-560.

23. Fukushima K, Fujii H, Yamamura T, Sugita A, Kameoka S, Nagawa H, Futami K, Watanabe T, Hatakeyama K, Sawada T, Yoshioka K, Kusunoki M, Konishi F, Watanabe M, Takahashi K, Ogawa H, Funayama Y, Hibi T, Sasaki I; Surgical Research Group, the Research Committee of Inflammatory Bowel Disease, Ministry of Health, Labour and Welfare of Japan. Pouchitis atlas for objective endoscopic diagnosis. J Gastroenterol 2007; 42: 799-806. 